\title{
La vida social de la energía: apuntes para el estudio territorializado de las transiciones energéticas
}

\section{Gustavo Blanco-Wells* (1)}

\section{Resumen}

Se presenta una propuesta teórica y metodológica para el estudio sociológico de la transición energética hacia energías renovables no convencionales, entendida como un proceso de cambio inducido vinculado a cambio climático, cuyos cursos de acción no están exentos de controversias sociotécnicas. La investigación se fundamenta en cuatro años de investigación sobre trayectorias territoriales de producción, uso y significación de la energía en tres regiones del sur-austral de Chile. A partir de una matriz teórica de la transición energética basada en los grados de renovabilidad y convencionalidad de la energía, se identifican diferentes regímenes sociotécnicos a partir de los cuales se debe indagar de modo empírico las formas situadas que adopta esta transición. Cada uno de estos regímenes fue investigado a través de casos de estudio lo que nos permite presentar aprendizajes generales de interés sociológico. A modo de conclusión, se propone ir más allá del concepto de régimen a través de la noción de ensamblaje, lo que permite explorar modos no lineales en que se asocian agentes heterogéneos en la territorialización de la energía. Además, se plantea que los grados de aceptación o rechazo social de la energía no debe ser el motor que mueva la investigación sobre esta materia, sino la forma en que las sociedades definen, debaten y construyen pragmáticamente formas democráticas y justas de uso energético en cada territorio. Desde nuestra perspectiva, esto supone avanzar en comprender estos procesos como parte de las soberanías energéticas de los pueblos.

Palavras-chave: Transición energética, Regímenes sociotécnicos, Ensamblaje, Territorialización, Soberanía energética.

* Universidad Austral de Chile, Valdivia, Chile. 


\section{The social life of energy: notes for the territorialized study of energy transitions}

\section{Abstract}

This paper presents a theoretical and methodological proposal for the sociological study of energy transition towards non-conventional renewable energy, as a process of induced transformation related to climate change, whose courses of action are not exempt from socio-technical controversies. The proposal is based on a four-year research program on territorial trajectories of production, use and making sense of energy in three regions of southern Chile. Based on a theoretical matrix of energy transition structured according the degrees of renewability and conventionality of energy, different socio-technical regimes are identified, from which the situated forms taken by this transition should be empirically investigated. Each of these regimes was researched through case studies, which allows us to present some general lessons of sociological interest. Concluding, it is proposed to transcend the concept of regime by means of the notion of assemblage, which allows for exploring non-linear ways heterogeneous agents team up in the territorialization of energy. In addition, we contend that the driving force behind research on this subject should not be the degrees of social acceptance or rejection of different sources of energy, but rather the way societies define, debate and pragmatically construct democratic and fair forms of energy use in a given territory. From our perspective, this means advancing in the understanding of these processes as part of people's energy sovereignty.

Keywords: Energy transition, Socio-technical regimes, Assemblage, Territorialization, Energy sovereignty.

\section{Introducción}

L ste artículo presenta una propuesta teórica y metodológica para lo que
se denomina la vida social de la energía ${ }^{1}$, entendida como el estudio
de los procesos territoriales, las prácticas organizativas, los usos, los significados y las disputas alrededor de la producción y el consumo de energía. Para ello, sitúa el análisis a partir de ciertas ilustraciones empíricas

\footnotetext{
1Esta denominación encuentra inspiración en el trabajo de Arjun Appadurai (1986) sobre "la vida social de las cosas", centrado en las relaciones que emergen a partir del intercambio y circulación de mercancías por parte de distintos actores sociales y grupos culturales. Appadurai inspiraría una serie de trabajos que comienzan desde el imperativo metodológico de revisar las condiciones de circulación de "objetos" como algo eminentemente social, es decir, "la vida social de las cosas" está constituida por y en un entramado de relaciones con propiedades emergentes que van más allá de su valor de uso e intercambio.
} 
del caso chileno que han surgido a través de un proceso de investigación de cuatro años. La idea de presentar una propuesta que revise el estatus sociológico de la energía surge en un escenario muy diferente de aquel de los primeros trabajos disciplinarios comprehensivos sobre el tema, como por ejemplo el tratado de Fred Cottrell, Energía y Sociedad (1955 [2009]), imbuidos en un claro enfoque evolucionario y bajo la impronta del ideario de la modernización y el desarrollismo. Ya en pleno siglo XXI, resulta inescapable enmarcar este debate en la importancia política que ha cobrado el desarrollo energético frente a la masiva evidencia de un cambio climático de origen antrópico (IPCC, 2014), en cuya base causal se encuentran sociedades con matrices de producción y consumo organizadas en torno a los combustibles fósiles (Giddens, 2011; Urry, 2011). En este escenario, las principales políticas de transición energética, asociadas al campo de la mitigación, aunque planteadas como un cambio de paradigma, están acopladas conceptualmente a un sistema eléctrico, crecimiento económico y estilos de vida modernos.

Para el caso de Chile, existen al menos dos nodos problemáticos en el cambio discursivo hacia una propuesta de transición energética: uno sociopolítico y otro académico. El primero se refiere a la noción de un "modelo energético" - que en la práctica se ha construido a partir de monopolios - concebido en torno a la electricidad, orientado al crecimiento urbano-industrial, estilos de vida modernos y estructurado en torno a criterios de mercado (Sohr, 2012). En este "modelo" de enfoque sectorial, las decisiones y acciones de producción, transmisión y consumo de energía son des-territorializadas y se constituyen en un campo disputado por actores sociales, incluso en aquellos proyectos de Energías Renovables No Convencionales (ERNC) que, aunque presentados como alternativas sustentables bajo una racionalidad tecnocrática, tienen altos niveles de conflicto territorial.

Un segundo nodo problemático es de corte teórico y está dado por los abordajes con que se ha estudiado el tema de la energía por parte de las ciencias sociales: los análisis están segmentados disciplinariamente, teñidos de un cierto determinismo energético y con escasa consideración por la materialidad, la técnica y la vida cotidiana. Los debates sobre energía en 
Chile han estado dominados por el mundo experto, con predominio de los enfoques ingenieriles y economicistas. Los aportes desde las otras ciencias sociales en Chile son bastante recientes (Cubillos; Estenssoro, 2011; Román, 2012; Susskind et al., 2014; Baigorrotegui; Parker; Estenssoro, 2014), pero con una producción en aumento entre las que se destaca la reciente contribución intelectual de un grupo de investigadores/as asociados al Núcleo Milenio de Investigación en Energía y Sociedad (Tironi; Sanazzaro, 2017; Boso; Ariztía; Fonseca, 2017; Ariztía; Boso; Tironi, 2017). Es solo a partir de la década de 2010 que las ciencias sociales en Chile comienzan a abordar la investigación sobre energía desde perspectivas centradas en los actores y sus prácticas, las experiencias territoriales y considerando el conjunto de tecnologías y materialidades propias de las diversas formas de energía, epistemologías y ontologías.

El Ilamado a los/las sociólogos/as iberoamericanos ${ }^{2}$ a desarrollar una sociología de la energía es bastante reciente (Pardo, 2006; Ariztía et al., 2017), algo que sí se encuentra de modo más vigoroso en agendas de investigación del mundo académico anglosajón (Rosa; Machlis; Keating, 1988; Shove, 1997; Henning, 2005; Jasanoff; Kim, 2013; Strauss; Rupp; Love, 2013, Sovacool, 2014). Avanzar en cubrir esta brecha de investigación en las ciencias sociales iberoamericanas nos permitirá comprender la multiplicidad de formas y flujos de la energía, la heterogeneidad de expresiones de producción y usos de acuerdo con los campos de acción y sitios sociales y, en último término, la organización social de la energía como diversidad de respuestas tecnológica, ecológica y ontológicamente diferenciadas.

En esta propuesta teórico-metodológica "la vida social de la energía" se despliega a través del estudio de su devenir en tres campos sociales entrelazados: a) la historia de la energía en el territorio; b) las políticas,

${ }^{2}$ El llamado a construir una sociología de la energía tiene un primer registro en idioma castellano en el texto de la académica española Mercedes Pardo (2006). De ahí que se considere como una propuesta iberoamericana, antes que exclusivamente latinoamericana. Por supuesto que más allá de lo idiomático, en esta propuesta es importante considerar las diferencias que se dan entre procesos y casos europeos y latinoamericanos de transición energética, además de la heterogeneidad interna nacional y dentro de los propios regímenes energéticos. 
proyectos y desarrollos territoriales en materia energética; y c) la experiencia fenomenológica de sus habitantes en el espacio doméstico y laboral. Estos campos constituyen lo que denomino trayectorias territoriales y serán explicados a lo largo del texto. Cabe señalar que el desarrollo de una agenda de investigación de esta naturaleza requiere de un enfoque interdisciplinario que integre a las distintas ciencias sociales con la ingeniería, la ecología, las humanidades e, idealmente, avance en prácticas transdisciplinarias que incluyan otras comunidades epistémicas. Solo desde esta pluralidad epistémica será posible comprender las trayectorias territoriales de producción, uso y significado de la energía desde la experiencia situada de los actores y evitar los efectos del determinismo implícito en muchas de las respuestas tecno-políticas contra el cambio climático.

El artículo está organizado en cuatro secciones: en primer lugar, se presentan los cambios gatillados por la transición energética vinculada a cambio climático y sus antecedentes en el "modelo" energético chileno; en segundo lugar, se presentan los contornos de una sociología de la energía; en tercer lugar, se desarrollan los alcances teóricos y metodológicos de la propuesta; para finalizar con algunas consideraciones generales.

\section{Cambio climático y transición energética}

El punto de partida de esta propuesta es el reconocimiento científico internacional del cambio climático antrópico (IPCC, 2014), devenido en discurso global e internalizado gradualmente en políticas públicas en los niveles nacionales y territoriales de Latinoamérica (Estenssoro, 2010; Postigo; Blanco; Chacón, 2013; Blanco; Fuenzalida, 2013). El reconocimiento de este fenómeno global de efectos locales va reorientando las propuestas de cambio social y tecnológico, ya no sólo en pos de mitigar el cambio climático, sino hacia la transformación de modos de producción, consumo y estilos de vida no sustentables, algo que se ha conceptualizado como Cambio Ambiental Global (O'Brien, 2012; ISSC, 2013; Blanco et al., 2017).

En el centro de estas transformaciones se encuentran las propuestas hacia una "transición energética" (Aitken, 2003; Verbong; Loorbach, 2012; Araújo, 2014), dado que se reconoce que el cambio climático es 
fundamentalmente un problema asociado a modos de producción industrial y estilos de vida organizados en base al uso intensivo de combustibles fósiles, principalmente carbón, petróleo y gas, todos fuentes no renovables de energía. En efecto, son los Gases de Efecto Invernadero (GEI), generados a partir de la industrialización con base en combustibles fósiles y "vidas con alta proporción de carbono" (Urry, 2011), lo que ha legitimado la idea de que las actividades antrópicas han puesto la vida de humanos y no-humanos en una situación de riesgo, expresada, en su dimensión más global, a través de un aumento sostenido de las temperaturas medias de la atmósfera, un fenómeno cuya velocidad no tiene precedentes en la historia de la humanidad (Weart, 2010; IPCC, 2014).

El cambio climático, como expresión de una crisis ambiental de escala planetaria, ha demandado esfuerzos de coordinación internacional y voluntad política para generar las transformaciones necesarias en modelos de desarrollo centrados en crecimiento industrial y actividades extractivas, altamente emisoras de GEI y propensas a deforestación y cambio de uso de suelo (Giddens, 2011). Evitar el cambio climático de causas antropogénicas es un discurso aceptado por casi todas las naciones y organizaciones multilaterales, en parte gracias al consenso en la interpretación de la información científica alcanzado en los informes del Panel Intergubernamental de Cambio Climático (PICC) y en los acuerdos de la Convención Marco de Naciones Unidas para el Cambio Climático (CMNUCC). En este escenario, el llamado a una transición energética ha permeado desde la esfera científica hasta la política y, a través de la construcción normativa de agendas, políticas y planes, cobra vida en el nivel nacional, para luego, a través de la materialización de proyectos que impulsan las ERNC, entrar al dominio de la vida cotidiana en el espacio territorial y local.

En este proceso, las propuestas de transición energética que se desarrollan en Chile tienen una trayectoria particular que pasamos a revisar para comprender el sentido de la propuesta analítica presentada en la siguiente sección. 


\section{Antecedentes de la transición energética en Chile: las contradicciones de una doble agenda}

En Chile, la discusión sobre energía se ha dado en los últimos 20 años, principalmente desde una perspectiva sectorial vinculada al mercado eléctrico y cruzada por diagnósticos que oscilan entre la fuerte dependencia de fuentes primarias externas (Wittelsbürger, 2007) hasta los sombríos y recurrentes análisis de "crisis energética" (Sohr, 2012). Esta sectorización económica de la producción y consumo de energía favorece los discursos y la construcción de evidencias desde una perspectiva económica que la vincula de manera lineal con el crecimiento económico (Cereceda; Errázuriz; Rivera, 2013).

Sin duda el proceso de modernización que comienza en la década de 1960 ha transformado la vida cotidiana de los/as chilenos/as, lo que se traduce en un aumento sostenido del consumo eléctrico, triplicándose desde 1990 a la década de 2010 (Cereceda et al., 2013). Este consumo de energía secundaria - obtenida mediante transformación de una fuente primaria - se reparte entre sector industrial y minero (40\%), seguidos por transporte (35\%) y finalmente el sector comercial, público y residencial (20\%) (Rainieri, 2018). Según este autor,

En el año 2015 los combustibles fósiles dan cuenta de un 70\% de la energía primaria que consume el país (petróleo crudo 28,5\%, importando cerca del 97\%; carbón $26,9 \%$, importando un $83 \%$; y gas natural $14,6 \%$, importando un $85 \%$ ), biomasa un $22,6 \%$ y que corresponde mayoritariamente a consumo de leña, hidroelectricidad $6,2 \%$, eólica $0,6 \%$, solar $0,3 \%$, y biogás por debajo de $0,3 \%$ (Rainieri, 2018, p. 10).

En cuanto al mercado eléctrico chileno, éste está constituido por actividades de generación, transmisión y distribución. La primera es operada bajo condiciones de mercado, mientras las otras dos son monopolios regulados que deben garantizar "libre acceso" a productores y consumidores (Guzowski; Recalde, 2010).

Los cambios políticos y jurídicos que dan origen al actual mercado eléctrico en Chile, basado en la privatización del sector, mínima regulación y libre competencia, se dan durante la dictadura de Pinochet con el DFL 
n¹ de 1982, que crea la Ley General de Servicios Eléctricos (LGSE). Entre 1998 y 1999 una pronunciada sequía afectó a parte importante de Chile, disminuyendo la cantidad de agua disponible en los embalses del centro y sur del país lo que provocó un programa de racionamiento eléctrico. A partir de este evento, ocurrido bajo el gobierno de Frei Ruiz-Tagle, comienza a gestarse la primera reforma a la LGSE, que se oficializa en 2004 bajo la presidencia de Lagos, mediante la Ley 19.940 (conocida como Ley Corta I) y luego, en 2008, a través de nuevos cambios con la Ley 20.018 (Ley Corta II). Este cambio en el marco legal del sector eléctrico chileno

en su origen no realizó una distinción normativa para las energías renovables no convencionales. Sin embargo, las modificaciones de la LGSE, oficializadas en marzo de 2004 (Ley 19.940), modificaron un conjunto de aspectos del mercado de generación eléctrica que afecta a todos los medios de generación, introduciendo elementos especialmente aplicados a las ERNC (Behnke; Estévez; Arias, 2009, p. 64).

Un cambio significativo para la apertura hacia una transición energética en Chile se aprecia en 2008 con la promulgación de la Ley 20.257 que, abiertamente, fomenta la generación de ERNC, pero no como una alternativa a las fuentes de energía convencional, sino en una relación de codependencia. Esto implica que, a partir de 2010, las empresas que tengan una capacidad instalada sobre los $200 \mathrm{Mw}$ deben incorporar las ERNC de modo que un $5 \%$ del total de la energía producida provenga de estas fuentes, porcentaje que debe incrementarse anualmente con la finalidad de alcanzar, en ese tiempo, la meta del 10\% para el 2024 (Behnke et al., 2009). En 2015 se oficializó la Política Energética de Chile 2050 (Ministerio de Energía, 2016), lo que se ha presentado como el inicio de una "revolución energética" (Pacheco, 2018), básicamente por un impulso decidido a las ERNC y porque incluye la participación ciudadana y el ordenamiento territorial en sus ejes discursivos, aun cuando sigue conceptualmente acoplada a un sistema eléctrico, al crecimiento económico y a la inversión privada, algo que algunos autores denominan hibridación neoliberal de la política eléctrica chilena (Maillet; Bugueño, 2019). 
Si bien el giro normativo general puede parecer auspicioso, pues marca el inicio de un escenario de transición energética hacia las ERNC, con tasas de incorporación en la matriz sin precedentes en Latinoamérica ${ }^{3}$, hay otro campo de políticas - las de cambio climático - que demuestran que el tema es bastante más complejo. En efecto, las contradicciones quedan en evidencia cuando consideramos los discursos en torno a las políticas de cambio climático expresados en el Acuerdo de París en 2015, según el cual Chile se compromete para el año 2030 a reducir sus emisiones de $\mathrm{CO} 2$ por unidad de PIB en un 30\% con respecto al nivel alcanzado en 2007, considerando un crecimiento económico futuro que le permita implementar las medidas para alcanzar este compromiso. En estas metas, la eficiencia energética, las energías renovables, el cambio de uso de suelo y los bosques tendrían un rol central en la estrategia. Si bien el crecimiento de las ERNC en la matriz ha sido sostenido, es el propio Ministerio del Medioambiente quien, en la Segunda Comunicación Nacional de Chile ante la CMNUCC, reconoce que "las emisiones del país están aumentando de manera importante, principalmente por el crecimiento del sector energía" (Ministerio del Medio Ambiente, 2011, p. 18). Asimismo, el análisis hecho por CEPAL (Vicuña, 2014) a los inventarios de GEI de quatro países reconoce que el aumento de emisiones del sector energía en Chile se debe, en gran medida, al crecimiento económico de la industria minera de alta importancia para el país (Vicuña, 2014). Aun cuando Chile no es un gran emisor de GEI, en 2011 fue el segundo país después de China con mayor crecimiento de emisiones, carbonización de la matriz sustentada en el crecimiento de termoeléctricas a carbón y en proyectos de explotación carbonífera a gran escala como los desarrollados en Isla Riesco, región de Magallanes (Yáñez; Garrido-Lepe, 2017).

\footnotetext{
${ }^{3}$ De acuerdo a datos de la Asociación de Energías Renovables de Chile (Acera), si en 2012 las ERNC tenían una potencia instalada de 952 MW, a diciembre de 2017 llegaron a 4.895 MWs. Es importante indicar que en 2010 la participación de las ERNC en la matriz eléctrica nacional bordeaba el 3,4\%, mientras que hacia finales de 2017 superó el 20\% de la generación (Fuente: Revista Electricidad, http://www.revistaei.cl/2018/01/09/2019-secumpliria-la-meta-20-ernc-fijada-ley-2025/.
} 


\section{Los caminos hacia las sociologías de la energía}

El campo teórico y empírico que la sociología ha ido construyendo en torno a la energía no es unívoco, ni en su decurso temporal, acrecentado por la centralidad de las acciones y políticas de cambio climático, ni en los agrupamientos temáticos y epistemológicos que lo constituyen en la actualidad. No hay aún una sociología de la energía, sino varios ámbitos temáticos que componen un campo cada vez más heterogéneo y especializado, tal como lo indican Ariztía y colaboradores (2017). El trabajo de Rosa, Machlis y Keating (1988) se encuentra entre los esfuerzos tempranos para sintetizar el campo sociológico de investigaciones que, hasta esa fecha, abordaban las relaciones entre energía y sociedad. Según los autores, en sus comienzos, la teoría se habría debatido en torno a las relaciones entre energía, tecnología y valores sociales, predominantemente en su vinculación con el progreso de las sociedades. El estudio teórico de estas relaciones presentaba variaciones importantes según el grado de determinación de la energía sobre la sociedad o viceversa. A partir de las décadas de 1960 y 1970, el auge de las preocupaciones medioambientales habría contribuido a la comprensión de la energía como un problema social, volviendo la mirada de los/as sociólogos/as hacia su capacidad de iluminar las relaciones entre los ecosistemas y los sistemas económicos y sociales. Así, la discusión se focalizó en las relaciones entre el crecimiento energético y el bienestar social, dando sustento a las políticas públicas en esta materia, principalmente de los países industrializados. En la década de 1990, los estudios en el ámbito de las políticas públicas han estado centrados en las opiniones y actitudes frente a los temas energéticos y en los impactos del desarrollo energético en términos sociales, culturales, demográficos, económicos y estéticos (Garniati et al., 2014), así como en los "imaginarios sociotécnicos" nacionales de las transiciones energéticas (Jasanoff; Kim, 2013).

Por otra parte, los enfoques microsociológicos ya cobraban fuerza a fines de la década de 1980, estimulados por la crisis ecológica y por la necesidad de demostrar la importancia de las ciencias sociales en el análisis de la vida cotidiana y la energía. En ellos se examinan los estilos de vida como factor determinante del consumo energético de los hogares, así como 
los elementos que inciden en la eficiencia de las acciones e inversiones realizadas por las personas y las empresas (Socolow, 1978; Wilhite et al., 2000; Hargreaves; Nye; Burguess, 2010; Shaw; Ozaki, 2013). La invisibilidad de la energía en la vida cotidiana y su escasa prioridad frente a otros temas medioambientales se convierte en el motivo de trabajo de Elizabeth Shove (1997), quien explora las miradas sociológicas a la energía y el medioambiente reconociendo dos agendas de investigación. Mientras una de ellas se plantea desde las creencias y prácticas individuales de los consumidores para ocuparse de las definiciones y formas de medición del consumo de energía, otra se propone abordar el consumo energético atendiendo a su organización social. Esta última, implica poner el énfasis en la construcción sociocultural de las preferencias y demandas de energía las cuales se encuentran encarnadas en infraestructuras tecnológicas, edificios, sistemas de transporte, desarrollo urbano y en modos de vida. Así, la autora destaca que lo que se logra visibilizar en una agenda, se vuelve invisible en la otra y viceversa.

Otro campo reciente es la literatura sobre conflictos ambientales en torno a proyectos energéticos. El impulso a la transición energética ha dado lugar a la expansión de las ERNC generando diversas respuestas sociales que, en algunos casos, han devenido en conflictos territoriales. Algunos autores, particularmente durante la década de 1990, han estudiado la oposición de los grupos locales a este tipo de proyectos desde la perspectiva del NIMBY (Not in my back yard) asumiéndolo como una reacción compleja que combina la desconfianza ante empresas e instituciones y el sentimiento de ser víctimas de un tratamiento injusto en la toma de decisiones. El concepto se ha utilizado para deslegitimar y desacreditar a los opositores locales clasificándolos en una postura egoísta, parroquial o ignorante (Burningham; Barnett; Thrush, 2006; Aitken, 2010; Zógrafos; Saladié, 2012). Frente a las limitaciones conceptuales del NIMBY, cierta academia europea giró, en la primera década de 2000, hacia el estudio de estos fenómenos bajo la etiqueta de la "aceptación social" de la energía (Wüstenhagen; Wolsink; Bürer, 2007; Batel; Devine-Wright; Tangeland, 2013), reconociendo con ello la existencia de articulaciones complejas entre políticas públicas, proyectos energéticos y territorios locales, así como la importancia de los 
espacios democratizadores que posibiliten la participación de los actores locales en las decisiones que los afectan.

En el caso de Chile, los conflictos territoriales o ambientales asociados a desarrollos energéticos se han documentado fundamentalmente en relación a proyectos de energías renovables convencionales, como la instalación de plantas hidroeléctricas, para distintos casos del sur del país como Ralco, Panguipulli y Aysén (Romero Toledo; Romero Aravena; Toledo, 2009; Román, 2012; Susskind et al., 2014; Tironi; Barandiarán, 2014; Höhl, 2018) y no convencionales como los parques eólicos de Chiloé (Kiritz; Durán; Montaña, 2015). También existe un reciente análisis sobre 26 conflictos ambientales vinculados a energía que incluyen otras fuentes convencionales como termoeléctricas (Maillet; Albala, 2018). Si bien los trabajos académicos publicados sobre conflictos energéticos son recientes y escasos, las controversias alrededor de estos proyectos se expresan vigorosamente a través de diversos movimientos sociales, los que, además de ejercer resistencia por medio de acción directa, comunican sus ideas a través de medios de divulgación ciudadanos o redes sociales.

\section{La vida social de la energía: fundamentos teóricos}

Proponer un examen a la vida social de la energía supone revisar algunos de los supuestos epistémicos y teóricos del tratamiento que las ciencias sociales han otorgado al tema. En primer lugar, supone una integración interdisciplinaria y un avance sistemático hacia la transdisciplina, incluyendo no sólo otros campos del conocimiento, sino otros actores sociales que participan de la construcción de saberes para la sustentabilidad. Este camino no es necesariamente conducente a la reducción de la incertidumbre, que es el rol asignado a la ciencia en los proyectos modernistas, pero sí a la integración de la complejidad dentro de los modos de aprensión de la realidad, lo que nos permite aportar grados de reflexividad a los procesos sociales (Funtowicz; De Marchi, 2000).

En segundo lugar, avanzar hacia el estudio de la vida social de la energía implica abandonar la sectorización económica de las actividades humanas y comprender que las relaciones sociales transcurren como parte de los procesos del habitar de un territorio, que se despliegan en un permanente 
devenir y no como procesos predeterminados (Ingold, 2000). Esta mirada del habitar territorial debe prestar atención no solo a los actores humanos, sino a los agentes no humanos, la materialidad y la tecnología, lo que se beneficia teóricamente de las corrientes socio-materialistas (De Landa, 2006; Latour, 2008; Ingold, 2011). En esta perspectiva la "construcción de lo social" no es un proceso meramente cognitivo y elaborado desde las percepciones individuales - y por lo tanto relativista - de la realidad, sino abordado desde una mirada relacional, que identifica las asociaciones establecidas entre actores humanos y no humanos, en un mundo donde lo social también está compuesto por materiales (Ingold, 2011), materialidades (Miller, 2005) y flujos (Umans; Arce, 2014). Contrarrestar las brechas en el conocimiento heredadas de una mirada sectorial, economicista, tecnocrática y antropocéntrica de la energía implica explorar aproximaciones multidimensionales centradas en las prácticas sociales, el efecto de la territorialización de procesos sociales en la constitución de nuevas entidades y ensamblajes (De Landa, 2006) y la heterogeneidad de respuestas de los agentes frente al cambio sociotécnico.

En esta propuesta, la vida social de la energía se despliega a través del estudio de su devenir en tres campos sociales entrelazados: a) la historia territorial, entendida como la historicidad de un proceso que ha tenido continuidades y discontinuidades que se deben trazar sincrónica y diacrónicamente; b) las políticas, proyectos y desarrollos territoriales en materia energética; entendida como un campo político disputado por la heterogeneidad de valores, representaciones y significados atribuidos a estos procesos por parte de agentes situados; c) la experiencia fenomenológica de sus habitantes en el espacio doméstico y laboral, dado que entendemos que la organización social de la producción, el consumo y la vida cotidiana genera formas diferenciadas de uso, significado y valoración de la energía que no suelen ser consideradas en los otros dos campos.

En esta propuesta, el concepto de trayectoria da cuenta de la inseparabilidad de estos tres campos para entender el devenir de los habitantes de una región o de territorios dentro de una región, en su particular relación con un dominio de la vida: la energía. El concepto de trayectoria, en este sentido, tiene un valor heurístico y se construye a partir 
del análisis integrado de los campos sociales arriba descritos en su dimensión procesual y no como eventos aislados de la vida social.

Junto al concepto de trayectoria, se vuelve relevante el de sistemas y regímenes sociotécnicos. El primero cobra importancia en los estudios sociales de ciencia y tecnología a través del historiador de la tecnología Thomas Hughes en su libro sobre la electrificación de occidente Networks of power (Hughes, 1983). El concepto de sistema sociotécnico enfatiza que, en el desarrollo de una tecnología, los aspectos de la organización social son inseparables de los componentes técnicos y materiales. Se introduce también como una forma de responder a los enfoques de determinismo tecnológico que privilegiaban interpretaciones en las que toda la organización social se explica a partir de un determinado desarrollo tecnológico. Aún más específico para los propósitos de esta propuesta es el concepto de regímenes sociotécnicos que "son configuraciones relativamente estables de instituciones, técnicas y artefactos, así como también regulaciones, prácticas y redes que determinan el desarrollo y uso 'normal' de tecnologías" (Rip y Kemp citados en Smith; Stirling; Berkhout, 2005, p. 1493). Este concepto es desarrollado por la escuela holandesa de estudios de transiciones tecnológicas, particularmente a partir de la perspectiva multinivel (Geels et al., 2007). Este enfoque teórico sostiene que las transiciones se producen a través de interacciones entre los procesos de tres niveles: (a) las innovaciones de nicho que constituyen el nivel micro donde emergen las novedades radicales, inicialmente configuraciones sociotécnicas bastante inestables; (b) cambios en el nivel del paisaje que es el ambiente exógeno (macroeconomía, patrones culturales etc.) que incide en los nichos y actores del régimen; y (c) la desestabilización del régimen que crea oportunidades para innovaciones específicas (Geels et al., 2007).

La idea de régimen tiene un rendimiento teórico importante cuando el punto de entrada es el estudio de procesos de normalización de tecnologías (políticas, normas, regulaciones, proyectos etc.). Sin embargo, su utilidad se reduce cuando lo que suponemos estable es sometido a tensiones por distintos agentes que contestan y reinterpretan esa normalización, abriendo espacio para nuevas configuraciones, más abiertas, flexibles e indeterminadas. En estos casos resulta más interesante la noción de 
ensamblaje, derivada de la concepción deleuziana de agenciamiento múltiple y heterogéneo. El ensamblaje va más allá del intento de capturar la composición inestable de las innovaciones tecnológicas, lo que Geels et al. (2007) denominan nicho de una transición, pues también incluye configuraciones no funcionales, transiciones inversas y puntos de fuga hacia arreglos sociomateriales impensados, más adecuados a la morfología rizomática e indeterminada de ciertos procesos.

En síntesis, la vida social de la energía propone el estudio de trayectorias sociotécnicas con la posibilidad de combinar o alternar entre regímenes y ensamblajes, de acuerdo al momento en que determinada forma energética comienza a ser territorializada.

\section{Alcances metodológicos de la propuesta}

Dada las múltiples formas en que se expresa la energía, esta propuesta se fundamenta metodológicamente en estudios de caso, idealmente extendidos, que examinen la vida social de regímenes sociotécnicos situados en territorios concretos y cercanos a la experiencia de las/los investigadores. La metodología debe orientarse a identificar, describir y analizar los procesos a través de los cuales la energía puede ser entendida como parte de la vida social de los territorios y elemento central de los conflictos y disputas de poder en torno a los recursos locales y sus naturalezas.

El estudio de caso extendido es una metodología en donde la experiencia de investigación vivida e información colectada a través de trabajo de campo se convierten en registro y/o datos que son analizados para períodos de tiempo extensos, identificando el reposicionamiento de los actores frente a diferentes eventos y situaciones, lo que permite enfatizar el carácter procesual del cambio social (Mitchell, [1956] 2006; Van Velsen, 1967; Burawoy, 1998). El estudio de caso extendido representa una herramienta de investigación social que tiene su origen en la Escuela de Antropología Social de Manchester (Evens; Handelman, 2006), aunque mantiene una reconocida afinidad epistemológica con la Escuela de Sociología de Chicago (Mills, [1956] 2006).

Los estudios de caso son una metodología flexible ya que a través de una serie de técnicas de recolección y registros de información de base 
etnográfica - observación, observación participante, historia oral, entrevistas, grupos focales, revisión documental y de archivos - permiten reconstruir e ilustrar algún aspecto de la vida social y abstraer conclusiones generales a través de un proceso de inferencia lógica y no estadística. Como lo define John Clyde Mitchell, un estudio de caso es "el material descriptivo básico que un observador ha ensamblado a través de diversos medios disponibles acerca de un fenómeno particular o serie de eventos con el fin explícito de elaborar conclusiones teóricas a partir de él" (Mitchell, [1956] 2006, p. 26). En el estudio de caso no opera la búsqueda de tipicalidad y representatividad, porque la unidad de análisis no es la "sociedad", la "cultura" o "grupos sociales" desde la que se extraen eventos que constituyen una muestra, sino los procesos sociales. Nuestra posibilidad de teorizar sobre esos eventos está dada por las relaciones lógicas establecidas entre categorías en un nivel abstracto, pero fundamentadas en una observación y descripción detallada del proceso estudiado.

\section{Territorializando la transición energética}

En la figura 1 se presenta una matriz que representa de modo sintético los preceptos teóricos de la transición energética implícitos en las políticas asociadas a cambio climático. Se trata de una expresión gráfica de aquellos aspectos teleológicos inscritos en los discursos del cambio sociotécnico planeado en materia energética. Por lo tanto, su valor es heurístico, no debe interpretarse como regímenes cerrados o monolíticos, ni tampoco como forma única de ordenar la relación entre sociedades, energía y cambio sociotécnico. Se trata de un punto de partida que debe contrastarse empíricamente con la realidad situada que se pretende estudiar. Conceptualmente, la figura plantea que la transición energética supone un desplazamiento tecnológico desde regímenes basados en carbono (combustibles fósiles) hacia el cuadrante superior derecho, o regímenes sustentables, una acción que, en teoría, es estimulada por políticas públicas e inversión en cambio tecnológico.

En esta propuesta se plantea la necesidad de explorar en profundidad casos que den cuenta de los distintos regímenes que integran cada cuadrante 
y sus eventuales desplazamientos a partir de intervenciones, proyectos $\mathrm{u}$ otros desarrollos energéticos. El movimiento lineal ascendente hacia la sustentabilidad y los supuestos consensos públicos sobre la renovabilidad y la convencionalidad deben ser empíricamente interrogados a partir de la diversidad de situaciones que pueden - o no - hacer posible y deseable una transición por parte de agentes territoriales en su relacionalidad con materialidades específicas.

Figura 1 - Matriz teórica de la transición energética.

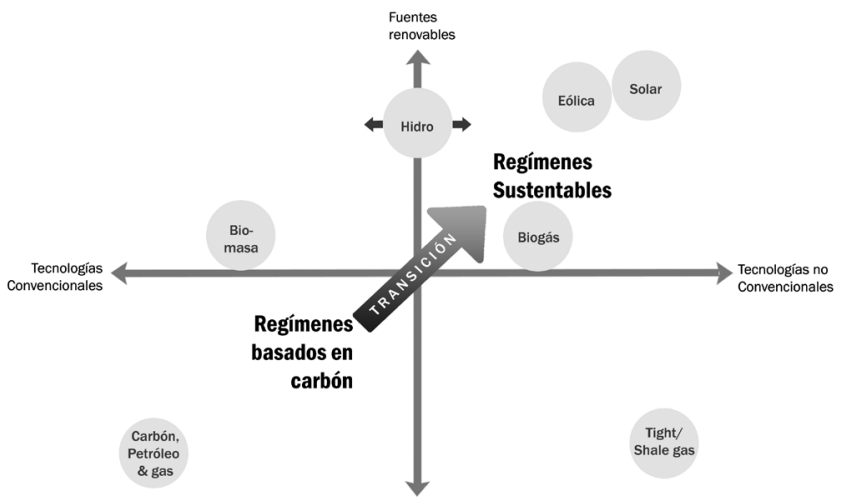

Fuente: elaboración propia.

Un hallazgo importante sobre esta matriz es que lo convencional de una tecnología, aunque parezca evidente, es exactamente eso: una convención social, un arreglo sujeto a variaciones de acuerdo a los cambios en las valoraciones y procesos de normalización de dicho régimen y cuyos límites se encuentran en permanente disputa. Por ejemplo, para el caso chileno, los proyectos de energía hidráulica son convencionales cuando superan los 20 Mw de capacidad de generación, una definición legal con consecuencias políticas, porque ello define además los mecanismos de evaluación de impactos a los que se someten los proyectos. Por lo tanto, que un proyecto de generación mini-hidro sea definido como no convencional 
y renovable, no disminuye el grado de conflictividad que puede presentar en su desarrollo, como ha sido anteriormente documentado para el caso de Chile. También los grados de renovabilidad de los recursos y fuentes energéticas de esta matriz se encuentran tensionados por debates y prácticas de valoración que van moviendo los límites de la "sustentabilidad", como por ejemplo en la importancia cultural de la leña (biomasa) como método de calefacción principal en el sur del país.

Como ya se ha destacado, la unidad heurística que agrupa los distintos tipos de experiencia sobre la energía es el de trayectoria territorial que, en cada caso, debería integrar tres campos sociales: la historia territorial, las políticas, proyectos y desarrollos territoriales y los modos de habitar. En este último campo se puede dar especial atención a incluir entre los criterios de selección de los casos el estudio de sistemas energéticos presentes en modos y medios de vida de relevancia para cada territorio o particularmente vulnerables a estas transformaciones: grupos sociales específicos, comunidades indígenas, campesinos/as etc.

En el trabajo de investigación desarrollado en Chile, desde 2015 a la actualidad, nos hemos enfocado en comprender la experiencia vivida por comunidades urbanas y rurales al tener que enfrentar el desarrollo de proyectos de energía renovable, tanto de tipo no convencional - parques eólicos, centrales mini-hidroeléctricas, biodigestores de gas, gasificación por fracturamiento - como convencional - transformaciones en el sistema de calefacción por leña y otros usos energéticos de la biomasa. También hemos identificado procesos de resistencia y controversias cuando se han llevado a cabo propuestas de transformación de energía convencional, por ejemplo, en relación al término del subsidio del gas en la región de Magallanes, el cierre de las minas de carbón en el centro sur de Chile y la construcción de proyectos de centrales hidroeléctricas de gran tamaño en las regiones de Los Ríos y Los Lagos, entre otros casos de estudio que nos han permitido documentar las variaciones de la transición energética.

Este conjunto de experiencias, cuyo detalle no es el propósito de este artículo, nos han enseñado que los cambios sociotécnicos se manifiestan en movimientos inesperados, lo que supone un cuestionamiento a la linealidad implícita en la idea de transición presentado en la matriz de la 
figura 1. Por ejemplo, el agotamiento de los pozos de gas convencionales en la Región de Magallanes y la amenaza de eliminación del subsidio que se otorga a la población para el uso cotidiano como energía térmica de este combustible, abrió paso a una movilización social masiva en la ciudad capital de esta región austral, lo que subsecuentemente permitió el desarrollo de la producción estatal de tight gas por vía de fracturamiento (no renovable, no convencional). Esta transición hacia una tecnología ambientalmente cuestionada no ha tenido oposición por parte de la comunidad regional, sino todo lo contrario, una aceptación de la extensión del programa estatal para explotación del gas.

Este y otros casos nos deben llevar también a reconsiderar el etnocentrismo implícito en la definición de la sustentabilidad que juzga como deseable un grupo de tecnologías e indeseables otras, sin considerar la historia material situada de los agentes que habitan los diversos territorios ni las posibilidades específicas para implementar esos cambios sin amenazar las actuales condiciones de existencia.

\section{Consideraciones finales}

La propuesta presentada en este artículo ha permitido que un grupo de investigación desarrolle estudios de mediano y largo plazo sobre la relación entre energía y sociedades territoriales frente a procesos de transiciones sociotécnicas, algo que he denominado "la vida social de la energía". Al mismo tiempo que identifica aportes de la sociología, particularmente a través de componentes metodológicos, en la práctica el estudio de estas transiciones se ha desarrollado a través de formas flexibles de investigación con orientación transdisciplinar en colaboración con colectivos sociales preocupados por la defensa del medio ambiente, los territorios y modos situados de desarrollo energético.

De particular interés para el estudio situado de estos procesos ha sido comprender que las transiciones no responden a un patrón histórico único, sino que son multidireccionales y surgen de asociaciones heterogéneas entre agentes y materialidades que las hacen posibles en condiciones específicas de existencia. Por lo tanto, el rendimiento teórico de los conceptos 
de trayectorias territoriales, regímenes sociotécnicos y ensamblajes va a depender del tipo de transición, el tipo de investigación y del despliegue espacio-temporal del equipo investigador.

En relación al rol de las ciencias sociales en el campo político, técnico y ciudadano de la transición energética, podemos señalar que el debate y las acciones se encuentran muy enmarcadas por los desafíos que impone el cambio climático a las sociedades. Alrededor de este fenómeno se ha formado una compleja trama de arreglos tecno-políticos que surgen desde los compromisos internacionales y que buscan viabilizar respuestas para disminuir sus efectos negativos. Dentro de estas respuestas, ha cobrado especial relevancia el impulso a diferentes expresiones de "transición" (energética, forestal, productiva, urbana). Una consideración abstracta de estos procesos podría llevarnos a pensar en transiciones lineales, susceptibles de ser programadas y con grados moderados o altos de aceptación social. Sin embargo, la investigación empírica que ha surgido a partir de la puesta en práctica de esta propuesta ha demostrado lo contrario. Las transiciones que se despliegan en la interfaz de medio ambiente y desarrollo, incluida la energética, son en muchos casos contestadas y debatidas por distintos grupos sociales territoriales, lo que demuestra los intereses divergentes entre estos colectivos y las élites tecno-políticas que participan de su planeamiento, inversión y desarrollo. Desde la perspectiva que surge de esta propuesta, debemos avanzar en entender estos procesos como parte de la soberanía energética de los pueblos. La aceptación o rechazo social de la energía no debe ser el motor que mueva la investigación sobre esta materia, sino la forma en que las sociedades definen, debaten y construyen formas democráticas y justas de uso energético en cada territorio. 


\section{Agradecimientos}

Este trabajo recibió aportes de la Comisión Nacional de Investigación Científica y Tecnológica de Chile a través de los Proyectos Fondecyt 1160857; Fondap 15150003; Fondap 15110009, así como de la Iniciativa Científica Milenio del Ministerio de Economía y Turismo del Gobierno de Chile.

Gustavo Blanco-Wells es Doctor en Sociología del Desarrollo y profesor asociado del Instituto de Historia y Ciencias Sociales de la Universidad Austral de Chile. También es Investigador Asociado del Núcleo Milenio Energía y Sociedad, del Centro de Investigación Dinámica de Ecosistemas Marinos de Altas Latitudes y del Centro de Ciencias del Clima y la Resiliencia, todos en Chile.

ఏ gblanco@uach.cl.

\section{Referencias}

1. AITKEN, Donald. Transitioning to a renewable energy future. White paper. Freiburg: International Solar Energy Society, 2003.

2. AITKEN, Mhairi. Why we still don't understand the social aspects of wind power: a critique of key assumptions within the literature. Energy Policy, v. 38, n. 4, p. 1834-1841, 2010. doi: 10.1016/j.enpol.2009.11.060.

3. APPADURAI, Arjun (Ed.). The social life of things: commodities in cultural perspective. Cambridge: Cambridge University Press, 1986.

4. ARAÚJO, Kathleen. The emerging field of energy transitions: progress, challenges, and opportunities. Energy Research \& Social Science, v. 1, p. 112121, 2014.

5. ARIZTÍA, Tomás; BOSO, Álex; TIRONI, Manuel. Sociologías de la energía. Hacia una agenda de investigación. Revista Internacional de Sociología, v. 75, n. 4, p. e074, 2017. doi: 10.3989/ris.2017.75.4.17.07.

6. BATEL, Susana; DEVINE-WRIGHT, Patrick; TANGELAND, Torval. Social acceptance of low carbon energy and associated infrastructures: a critical discussion. Energy Policy, v. 58, p. 1-5, 2013. doi: 10.1016/j.enpol.2013.03.018.

7. BAIGORROTEGUI, Gloria; PARKER, Cristian; ESTENSSORO, Fernando. Visiones sobre los tránsitos socio-técnicos hacia patrones de consumo sustentable en agua y energía en la minería sudamericana - dos controversias argentinas. Sociologias, v. 16, n. 37, p. 72-111, 2014. doi: 10.1590/15174522-016003704.

8. BEHNKE, Rodrigo P.; ESTÉVEZ, Guillermo J.; ARIAS, Ignacio A. Las energías renovables no convencionales en el mercado eléctrico chileno. Santiago de Chile: Proyecto Energías Renovables No Convencionales (CNE/GTZ), 2009.

9. BLANCO, Gustavo; FUENZALIDA, María I. La construcción de agendas científicas sobre cambio climático y su influencia en la territorialización de políticas 
públicas: reflexiones a partir del caso chileno. In: POSTIGO, Julio (Ed.). Cambio climático, movimientos sociales y políticas públicas. Santiago: CLACSO/ICAL, 2013, p. 75-102.

10. BLANCO, Gustavo; GÜNTER Griselda; GUTIÉRREZ, Ricardo; VALENCIA, Javier. Introducción. Cambio ambiental global y políticas ambientales en América Latina. In: GÜNTER, Griselda; GUTIÉRREZ, Ricardo (Coord.). La política del ambiente en América Latina. Una aproximación desde el cambio ambiental global. 2017. p.15-36.

11. BOSO, Álex; ARIZTÍA, Tomás; FONSECA, Francisca. Usos, resistencias y aceptación de tecnologías energéticas emergentes en el hogar. El caso de la política de recambio de estufas en Temuco. Revista Internacional de Sociología, v. 75, n. 4, p. e078, 2017. doi: 10.3989/ris.2017.75.4.17.04.

12. BURNINGHAM, Kate; BARNETT, Julie; THRUSH, Diana. The limitations of the NIMBY concept for understanding public engagement with renewable energy technologies: a literature review. Manchester: School of Environment and Development, University of Manchester, 2006.

13. BURAWOY, Michael. The extended case method. Sociological Theory, v. 16, n. 1, p. 4-33, 1998. doi: 10.1111/0735-2751.00040.

14. CERECEDA, Pilar; ERRÁZURIZ, Ana María; RIVERA, Juan. Energía. La electricidad en un mundo que avanza. Santiago de Chile: Pontificia Universidad Católica de Chile, 2013.

15. COTTRELL, Fred. Energy \& Society. The relation between energy, social change, and economic development. Bloomington: AuthorHouse, 2009.

16. CUBILLOS, Adela; ESTENSSORO, Fernando (Comp.). Energía y medio ambiente. Una ecuación difícil para América Latina. Santiago de Chile: Instituto de Estudios Avanzados, Universidad de Santiago, 2011.

17. DE LANDA, Manuel. A new philosophy of society. Assemblage theory and social complexity. Londres: Continuum, 2006.

18. ESTENSSORO, Fernando. Crisis ambiental y cambio climático en la política global: un tema crecientemente complejo para América Latina. Revista Universum, v. 2, n. 25, p. 57-77, 2010. doi: 10.4067/S0718-23762010000200005.

19. EVENS, Terry; HANDELMAN, Don (Eds). The Manchester school: practice and ethnographic praxis in Anthropology. Oxford, NY: Berhahn Books, 2006.

20. FUNTOWICZ, Silvio; DE MARCHI, Bruna. Ciencia posnormal, complejidad reflexiva y sustentabilidad. In: LEFF, Enrique (Coord.). La complejidad ambiental. Ciudad de México: Siglo XXI/PNUMA, 2000. p. 54-84.

21. GARNIATI Leuserino; OWEN Alan; KRUIJSEN, Joanneke; ISHADAMY, Jakob; WIBISONO Ilarius. Interface between appropriate technology and sustainable energy policy in vulnerable societies. Sustainable Cities and Societies, v. 12, p. 9-15, 2014. doi: 10.1016/j.scs.2013.10.003.

22. GEELS, Frank: SCHOT, Johan. Typology of sociotechnical transition pathways. Research Policy, v. 36, n. 3, p. 399-417, 2007. doi: 10.1016/j.respol.2007.01.003. 
23. GIDDENS, Anthony. Politics of climate change. 2nd Ed. Cambridge: Polity Press, 2011.

24. GUZOWSKI, Carina; RECALDE, Marina Y. Latin American electricity markets and renewable energy sources: the argentinean and chilean cases. International Journal of Hydrogen Energy, v. 35, n. 11, p. 5813-5817, 2010. doi: 10.1016/j. ijhydene.2010.02.094.

25. HARGREAVES, Tom; NYE, Michael; BURGUESS Jacquelin. Making energy visible: a qualitative field study of how householders interact with feedback from smart energy monitors. Energy Policy, v. 38, n. 10, p. 6111-6119, 2010. doi: 10.1016/j.enpol.2010.05.068.

26. HENNING, Annete. Climate change and energy use. Anthropology Today, v. 21, p. 8-12, 2005. doi: 10.1111/j.0268-540X.2005.00352.x.

27. HÖHL, Johanna. Hidroelectricidad y pueblos indígenas: un análisis del megaproyecto Ralco en la región Bío Bío, Chile. In: ULLOA, Astrid; ROMEROTOLEDO, Hugo (Eds). Agua y disputas territoriales en Chile y Colombia. Bogotá: Universidad de Colombia, 2018. p. 297-331.

28. HUGHES, Thomas. Networks of power: electrification in western society 1880 - 1930. Baltimore; Londres: The Johns Hopkins University Press, 1983.

29. INGOLD, Tim. The perception of the environment. Essays in livelihood, dwelling and skill. Londres; Nueva York: Routledge, 2000.

30. INGOLD, Tim. Being alive. Essays on movement, knowledge and description. Londres; Nueva York: Routledge, 2011.

31. IPCC. Summary for policymakers. In: FIELD, Cristopher et al. (Eds.). Climate change 2014: impacts, adaptation, and vulnerability. Part A: Global and Sectoral Aspects. Contribution of Working Group II to the Fifth Assessment Report of the Intergovernmental Panel on Climate Change. Cambridge; Reino Unido; Nueva York: Cambridge University Press, 2014. p. 1-32.

32. ISSC - International Social Science Council; UNESCO - United Nations Educational, Scientific and Cultural Organization. World social science report 2013: changing global environments. Paris: OECD y Unesco, 2013.

33. JASANOFF, Sheila; KIM San H. Sociotechnical imaginaries and national energy policies. Science as Culture, v. 22, n. 2, p. 189-196, 2013. doi: 10.1080/09505431.2013.786990.

34. KIRITZ, Gabriel; DURÁN Vanesa; MONTAÑA, Álvaro. Ordenamiento territorial energético en Chile: legitimización social de la matriz energética nacional e impactos en el Archipiélago de Chiloé. Boletín del Patrimonio Natural de Chiloé, v. 1, n. 2, p. 3-21, 2015. Disponible en: http://naturalcapitalproject. stanford.edu/pubs/Kiritz_OTE.pdf.

35. LATOUR, Bruno. Reensamblar lo social. Una introducción a la teoría del actor-red. Buenos Aires: Manantial, 2008. 
36. MAILLET, Antoine; ALBALA, Adrián. Conflictos socioambientales en los proyectos eléctricos en Chile (2005-2016). Un análisis configuracional. América Latina Hoy, v. 79, p. 125-149, 2018. doi: 10.14201/alh201879125149.

37. MAILLET, Antoine; BUGUEÑO, Joaquín. Hibridación de las políticas neoliberales. El caso de la reforma a la política eléctrica en Chile (2014-2016). Gestión y Política Pública, v. 38, n. 1, p. 207-235, 2019. doi: 10.29265/gypp. v28i1.546.

38. MILLER, Daniel (Ed). Materiality. Durham, Estados Unidos: Duke University Press, 2005.

39. MILLS, David. Made in Manchester? Methods and myths in disciplinary history. In: EVENS, Terry, M. S.; HANDELMAN, Don (Eds). The Manchester school: practice and ethnographic praxis in anthropology. Nova York: Berghahn Books, 2006 [1956]. p. 165-179.

40. MINISTERIO DE ENERGIA DE CHILE. Energía 2050 política energética de Chile. Santiago de Chile: Ministerio de Energía, 2016.

41. MINISTERIO DEL MEDIO AMBIENTE DE CHILE. Segunda comunicación nacional ante la Convención Marco de las Naciones Unidas sobre Cambio Climático. Santiago de Chile: Ministerio del Medio Ambiente, 2011.

42. MITCHELL, John C. Case and situation analysis. In: EVENS, Terry M. S.; HANDELMAN, Don (Eds). The Manchester school: practice and ethnographic praxis in anthropology. Nova York: Berghahn Books, 2006 [1956]. p. 23-45.

43. O'BRIEN, Karen. Global environmental change II: from adaptation to deliberate transformation. Progress in Human Geography, v. 36, n. 5, p. 667676, 2012. doi: 10.1177/0309132511425767.

44. PACHECO, Máximo (Ed.). Revolución energética en Chile. Santiago de Chile: Ediciones UDP, 2018.

45. PARDO, Mercedes. Hacia una sociología de la energía. Cuadernos de Energía, v. 11, p. 16-20, 2006. doi: 10.1177\%2F0309132511425767.

46. POSTIGO, Julio; BLANCO, Gustavo; CHACÓN Pablo. Social sciences at the crossroads: global environmental change in Latin America and the Caribbean. In: ISSC/UNESCO. World social science report 2013: changing global environments. Paris: OECD Publishing; UNESCO Publishing, 2013. p. 142-151.

47. RAINIERI, Ricardo. Transición energética en Chile: una verdad incómoda. CLAPES UC. Documentos de Trabajo n. 39, 5 ene. 2018.

48. ROMÁN, Jaime. Energía, territorios y poblaciones indígenas: análisis retrospectivo del megaproyecto hidroeléctrico Ralco. Revista Conservación Ambiental, v. 2, n. 1, p. 37-42, 2012.

49. ROMERO TOlEDO, Hugo; ROMERO ARAVENA, Hugo; TOlEDO Ximena. Agua, poder y discursos en el conflicto socio-territorial por la construcción de represas hidroeléctricas en la Patagonia Chilena. Anuario de Estudios Americanos. Madrid, v. 66, n. 2, p. 81-103, 2009. Disponible en: http://repositorio.uchile.cl/ handle/2250/117821. 
50. ROSA, Eugene; MACHLIS, Gary; KEATING, Keneth. Energy and society. Annual Review of Sociology, v. 14, p. 149-172, 1988. doi: 10.1146/annurev. so.14.080188.001053.

51. SHAW, Isabel; OZAKJ, Ritsuko. Energy provision and housing development: re-thinking professional and technological relations. Energy Policy, v. 60, p. 427430, 2013. doi:10.1016/j.enpol.2013.04.060.

52. SHOVE, Elizabeth. Revealing the invisible: sociology, energy and the environment. In: REDCLIFT, Michael; WOODGATE, Graham (Eds). The international handbook of environmental sociology. Cheltenham: Edward Elgar, 1997. p. 261-273.

53. SMITH, Adrian; STIRLING, Andy; BERKHOUT, Frans. The governance of sustainable socio-technical transitions. Research Policy, v. 34, p. 1491-1510, 2005. doi: 10.1016/j.respol.2005.07.005.

54. SOCOLOW, Red (Ed). Saving energy in the home. Princeton's experiments at Twin Rivers. Center for environmental studies. Cambridge, MA: Ballinger Publishing Company, 1978.

55. SOHR Raúl. Chile a ciegas. La triste realidad de nuestro modelo energético. Santiago de Chile: Random House Mondadori, 2012.

56. SOVACOOL, Benjamin. What are we doing here? Analyzing fifteen years of energy scholarship and proposing a social science research agenda. Energy Research \& Social Science, v. 1, p. 1-29, 2014. doi: 10.1016/j.erss.2014.02.003

57. STRAUSS, Shara; RUPP, Stephanie; LOVE, Thomas (Eds.). Cultures of energy: power, practices, technologies. Nueva York; Walnut Creek, CA: Left Coast Press, 2013.

58. SUSSKIND, Lawrence; KAUSEL, Teodoro; AYLWIN, José; FIERMAN, Elizabeth. The future of hydropower in Chile. Journal of Energy \& Natural Resources Law, v. 32, n. 4, p. 425-481, 2014. doi: 10.1080/02646811.2014.11435370

59. TIRONI, Manuel; BARANDIARAN, José. Neoliberalism as political technology: expertise, energy and democracy in Chile. In: MEDINA, Eden; COSTA MÁRQUES, Ivan; HOLMES, Christina (Eds.). Beyond imported magic: studying science and technology in Latin America. Cambridge, MA: MIT Press, 2014. p. 305-329. doi:10.7551/mitpress/9780262027458.003.0015.

60. TIRONI, Manuel; SANNAZZARO, Jorgelina. Energía huilliche. Experimentos en integración y disensos ontológicos en un parque eólico. Revista Internacional de Sociología, v. 75, n. 4, e080, 2017: doi: 10.3989/ris.2017.75.4.17.06

61. UMANS, Laurent; ARCE, Alberto. Fixing rural development cooperation? Not in situations involving blurring and fluidity. Journal of Rural Studies, v. 34, p. $337-$ 344, 2014. doi: 10.1016/j.jrurstud.2014.03.004

62. URRY, John. Climate change and society. Cambridge: Polity Press, 2011.

63. VAN VELSEN, Jaap. The extended-case method and situational analysis. In: EPSTEIN, A. L. (Ed.) The craft of social anthropology. Londres: Tavistock Publications, 1967. p. 129-153. 
64. VERBONG, Geert; LOORBACH, Der (Eds.). Governing the energy transition. Reality, illusion or necessity? Nueva York: Routledge, 2012.

65. VICUÑA, Sebastián. Inventarios de emisiones de gases de efecto invernadero. Un análisis para Chile, El Salvador, México y el Uruguay. Serie estudios del cambio climático en América Latina. Santiago de Chile: Comisión Económica para América Latina y el Caribe (CEPAL), Naciones Unidas, 2014.

66. WEART, Spencer. The idea of anthropogenic global climate change in the 20th Century. WIREs: Climate Change, v. 1, n. 1, p. 67-81, 2010. doi: 10.1002/wcc.6.

67. WILHITE Harold; SHOVE Elizabeth; LUTZENHISER, Loren; KEMPTON, Willett. Twenty years of energy demand management: we know more about individual behavior but how much do we really know about demand. In: American Council for an Energy-Efficient Economy, 2000, Pacific Grove. Proceedings [...]. Washington: ACEEE, p. 435-453.

68. WITTELSBÜRGER, Helmut. La política energética de Chile: de la dependencia al desarrollo sostenible. El futuro es de las energías renovables. Diálogo Político, v. 4, p. 35-52, 2007.

69. WÜSTENHAGEN, Rolf; WOLSINK, Maarten; BÜRER, MaryJ. Social acceptance of renewable energy innovation: an introduction to the concept. Energy Policy, $\mathrm{V}$. 35, n. 5, p. 2683-2691, 2007. doi: 10.1016/j.enpol.2006.12.001

70. YÁÑEZ, César; GARRIDO-LEPE, Martín. El tercer ciclo del carbón en Chile, de 1973 a 2013: del climaterio al rejuvenecimiento. América Latina en la Historia Económica, v. 24, n. 3, p. 224-258, 2017. doi: 10.18232/alhe.v24i3.833

71. ZÓGRAFOS Christos; SALADIÉ, Sergi. La ecología política de conflictos sobre energía eólica. Un estudio de caso en Cataluña. Documentsd'Anàlisi Geogràfica, v, 58, n. 1, p. 177-192, 2012. doi: 10.5565/rev/dag.202 